\title{
Clinical Application of Vibration Controlled Transient Elastography in Patients with Chronic Hepatitis B
}

\author{
Xie-Er Liang and Yong-Peng Chen* \\ State Key Laboratory of Organ Failure Research, Guangdong Provincial Key Laboratory of Viral Hepatitis Research, Department of \\ Infectious Diseases and Hepatology Unit, Nanfang Hospital, Southern Medical University, Guangzhou, China
}

\begin{abstract}
Evaluation of the extent and progression of liver fibrosis and cirrhosis is of critical importance in the management and prognosis of patients with chronic hepatitis B. Due to the limitation of liver biopsy, non-invasive methods, especially liver stiffness measurement (LSM) by vibration controlled transient elastography, have been developed and widely applied for liver fibrosis assessment. LSM aims to reduce, but not to substitute, the need for liver biopsy for fibrosis/ cirrhosis diagnosis. While LSM may have potential utility in monitoring treatment response, its applications in prediction of liver complications in terms of portal hypertension and esophageal varices, as well as disease prognosis, have been gradually validated. Here, we review the latest clinical applications of LSM in patients with chronic hepatitis B.

Citation of this article: Liang XE, Chen YP. Clinical application of vibration controlled transient elastography in patients with chronic hepatitis B. J Clin Transl Hepatol 2017;5 (4):368-375. doi: 10.14218/JCTH.2017.00006.
\end{abstract}

\section{Introduction}

Hepatitis B virus (HBV)-related fibrosis or cirrhosis is a progressive disease, ultimately resulting in end-stage liver disease or hepatocellular carcinoma (HCC) and accounting for over one million deaths per year worldwide. ${ }^{1-4}$ Evaluation of the extent and progression of liver fibrosis and of the risk of cirrhosis, therefore, plays an important role in the management and prognosis of patients with chronic hepatitis $B$ $(\mathrm{CHB})$. In the management of $\mathrm{CHB}$, the two clinically relevant endpoints for staging liver fibrosis are: first, detection of significant fibrosis (METAVIR $F \geq 2$ or Ishak $\geq 3$ ), which indicates that patients should receive antiviral treatment; and, second, detection of cirrhosis (METAVIR F4 or Ishak 5-6), which indicates not only the potential for prescribing long-term antiviral therapy but also monitoring for complications related to portal hypertension and regular screening for HCC.

Keywords: Hepatitis B; Liver fibrosis; Liver stiffness; Transient elastography. Abbreviations: ALT, alanine aminotransferases; AUROC, area under the receiver operating characteristic curve; $\mathrm{CHB}$, chronic hepatitis $\mathrm{B}$; HBV, hepatitis B virus; HCC, hepatocellular carcinoma; HVPG, hepatic venous pressure gradient; LSM, liver stiffness measurement; NLR, negative likelihood ratio; PLR, positive likelihood ratio; ULN, upper limit of normal; VCTE, vibration controlled transient elastography.

Received: 18 January 2017; Revised: 28 May 2017; Accepted: 26 June 2017

*Correspondence to: Yong-Peng Chen, Department of Infectious Diseases, Nanfang Hospital, Southern Medical University, Guangzhou 510515, China. Tel: +86-20-62787432, Fax: +86-20-62786530, E-mail: cyp@smu.edu.cn
Liver biopsy has been the "gold standard" for liver fibrosis staging for decades. However, it is hampered by its invasive nature, risk of complications and patient discomfort. ${ }^{5}$ In addition, sampling error could result in underestimation of liver fibrosis and false negative diagnosis of cirrhosis (in $10 \%-30 \%$ of cases). ${ }^{6}$ To address these issues, non-invasive methods have been developed and validated for liver fibrosis assessment, among which liver stiffness measurement (LSM) by vibration controlled transient elastography (VCTE) is one of the most promising techniques. Besides staging fibrosis, LSM has been demonstrated to have potential utility in monitoring treatment response and surveillance of liver-related events. $^{7}$

This article reviews the clinical application of VCTE in patients with $\mathrm{CHB}$ and discusses the points and prospects to be considered when using VCTE for the management of CHB.

\section{Assessing significant fibrosis}

Like other non-invasive methods, when interpreting the diagnostic performance of VCTE, several methodological problems should always be kept in mind. ${ }^{7,8}$ Application of the imperfect gold standard of liver biopsy as the reference for assessment of diagnostic accuracy of LSM reduces the potential to reach optimal diagnostic accuracies assessed using the area under the receiver operating characteristic curve (AUROC) of $>0.9 .{ }^{9}$ Therefore, an AUROC of 0.85-0.90 may be considered as highly accurate. On the other hand, direct comparisons of AUROCs and their related optimal diagnostic cutoffs derived from two specific populations is usually not suitable, as the spectrum effects of the population should be taken into consideration.

Prevalence of the disease among the investigated population also plays a role in the diagnostic performance, impacting the predictive value especially, of a non-invasive method. For the clinical application of LSM in staging fibrosis, it is rational to reduce the need of liver biopsy but not to substitute this gold standard. ${ }^{10} \mathrm{~A}$ likelihood ratio, which is independent of disease prevalence, of $>10$ or $<0.1$ used in cutoff determination is strong enough to confirm or exclude a diagnosis. ${ }^{11}$ Accordingly, only the residual patients with LSM falling within the so-called grey zone (i.e. LSM lower than the confirming cutoff and higher than the excluding cutoff) need liver biopsies (Fig. 1).

Determination of the stage of liver disease is important in guiding antiviral therapy decisions and the need for surveillance. In terms of guiding antiviral therapy, differentiation of significant fibrosis (METAVIR $F \geq 2$ or Ishak $\geq 3$ ) from mild fibrosis (METAVIR $F<2$ or Ishak $<3$ ) has critical clinical 


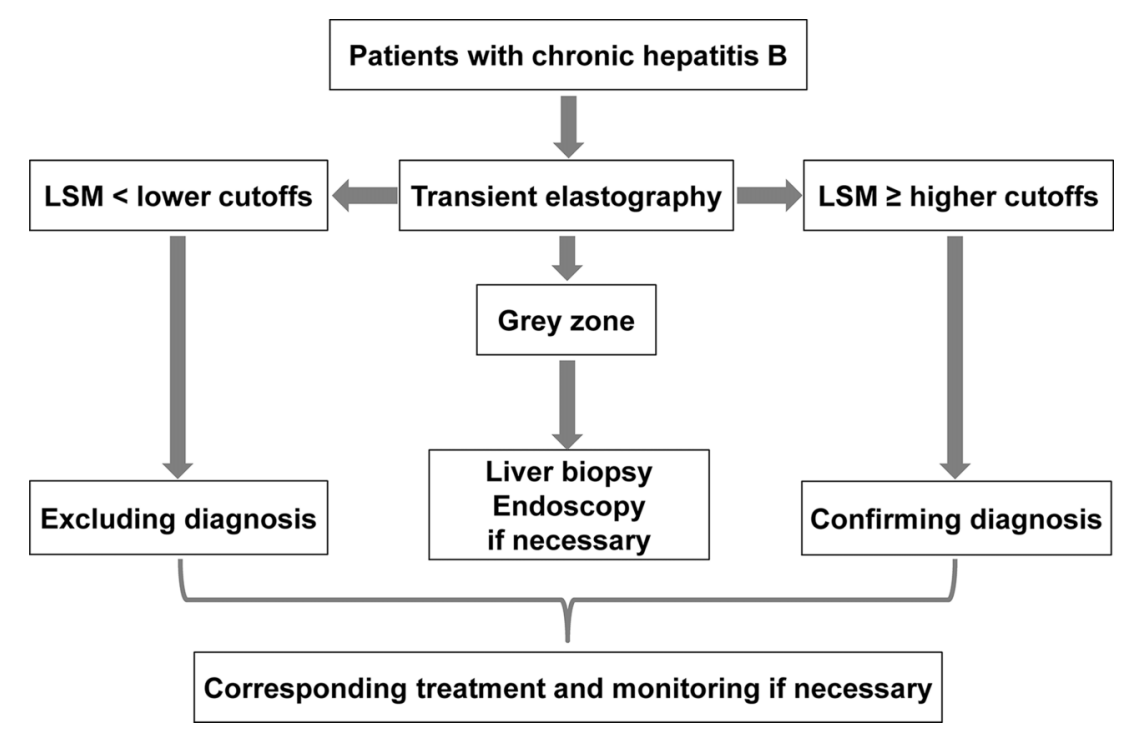

Fig. 1. Algorithm and schematic diagram for the adjuvant application of liver stiffness measurement (LSM) by vibration controlled transient elastography for non-invasive diagnosis of liver fibrosis/cirrhosis and portal hypertension in patients with chronic hepatitis B.

implications for initiation, especially for patients over the age of 30 years, with intermediate elevated alanine aminotransferase (ALT; i.e. $<2$ times the upper limit of normal (ULN)) and high HBV DNA levels. Therefore, determining the absolute stage of fibrosis is less important than determining whether patients have advanced liver disease with fibrosis METAVIR $F \geq 2$ or Ishak $\geq 3$.

The performance of LSM in detecting significant fibrosis is inferior to that for cirrhosis, with AUROC 0.66-0.87 for significant fibrosis (Table 1). Among the suggested cutoffs for detecting significant fibrosis, only cutoffs by Jia et al. ${ }^{12}$ Chen et al. ${ }^{13}$ and Vigano et al. ${ }^{14}$ were characterized with negative likelihood ratio (NLR) of nearly 0.1 or positive likelihood ratio (PLR) of nearly 10.0 , which could determine significant fibrosis with enough strong statistical evidence. Considering the lack of relevant clinical consequences of false negative cases and the considerable costs of antivirus treatment of false positive cases, it is recommended that the confirming diagnosis of significant fibrosis may be of more value for clinical practice. Thus, LSM of $9.4 \mathrm{kPa}$ (PLR of 14.0) and of $9.8 \mathrm{kPa}$ (PLR of 11.0) could be selected as confirming diagnosis cutoffs, with the latter derived from a large cohort but a larger biopsy sample study may be superior.

It has been reported that hepatitis flares may affect LSM results; therefore, serum levels of ALT should always be taken into account when interpreting results from VCTE. ${ }^{15}$ To avoid the risk of false positive diagnosis, certain investigators have suggested that LSM cutoffs should be adjusted according to ALT levels. ${ }^{16,17}$ However, a study of large biopsy samples indicated that ALT level exerted influenced on cutoffs for detecting advanced fibrosis but not significant fibrosis. ${ }^{13}$ Regarding the purpose of guiding antiviral therapy, LSM use is preferred in patients with normal ALT or intermediate elevated ALT $\left(<2\right.$ ULN). ${ }^{18}$ There have been studies reporting that LSM could be used as a supplemental tool to HBV DNA, to follow inactive carriers or to better identify patients who may have ongoing disease activity or significant fibrosis and who require liver biopsy. ${ }^{18,19}$ A recent study also suggested a combination of $\mathrm{HBV}$ DNA $\leq 2000 \mathrm{IU} / \mathrm{mL}$ and $\mathrm{LSM} \leq 6.2 \mathrm{kPa}$ to detect inactive HBV carriers with positive predictive value of $98.5 \%$ in a single time point evaluation. ${ }^{20}$

\section{Detecting cirrhosis}

Current nucleos(t)ide treatment of hepatitis B is not curative, and may generally be lifelong for patients with liver cirrhosis. Besides, cirrhotic patients are subject to development of subsequent complications and need intensive surveillance for development of HCC. Thus, non-invasive methods to identify patients with cirrhosis must have high sensitivity, to reduce the risk of false negatives, as well as high specificity, to avoid diagnostic errors resulting in increased economic burden of long-term surveillance of the cirrhotic complication. LSM has proven potent accuracy for cirrhosis diagnosis, with AUROC $0.80-0.97$ and suggestive cutoff of $8.4-29.2$ (Table 2).

LSM $11.6 \mathrm{kPa}$ was suggested by a large cohort study ( $n=$ $567)$ with sufficient biopsy sample size $(\geq 15 \mathrm{~mm})$ from Korea, characterized with NLR of 0.20 and PLR of $5.70 ;{ }^{21}$ these findings implied that cutoff for confirming diagnosis should be far higher than $11.6 \mathrm{kPa}$ and, therefore, the cutoff for excluding diagnosis should be slightly lower than $11.6 \mathrm{kPa}$. Another large cohort study $(n=469)$ from China may be criticized by its inclusion of patients with insufficient biopsy sample size (lower than $15 \mathrm{~mm}),{ }^{12}$ which would have impaired confidence of the findings from the "gold standard" liver biopsy. In a study from India, the reported suggested cutoff for cirrhosis may be unreliable, due to the low prevalence of cirrhosis (5.9\%). ${ }^{22}$

For cutoffs determining cirrhosis, the suggested LSMS ranging between $11.8 \mathrm{kPa}$ and $18.5 \mathrm{kPa}$ were characterized with PLR of $>10.0$. The LSM of $18.5 \mathrm{kPa}$ with PLR of 15.2 suggested by Liang et al. ${ }^{23}$ and the LSM of $18.2 \mathrm{kPa}$ with PLR of 19.0 suggested by Marcellin et al. ${ }^{24}$ implied that the rational cutoff for ruling in diagnosis should be lower than $18.2 \mathrm{kPa}$. While cutoffs of $13.4 \mathrm{kPa}$ and $13.1 \mathrm{kPa}$ were derived from study cohorts of nearly 100 patients, cutoffs of $16.9 \mathrm{kPa}$ and $17.0 \mathrm{kPa}$ were suggested by study cohorts with 
Liang X.E. et al: VCTE in chronic hepatitis B

Table 1. Diagnostic performance of VCTE for significant fibrosis (METAVIR F $\geq 2$ ) in patients with chronic hepatitis B

\begin{tabular}{|c|c|c|c|c|c|c|c|c|c|}
\hline Author & Country/year & Patients, $n$ & $F \geq 2, \%$ & Cutoff kPa & AUROC & $\mathrm{Se}, \%$ & NLR & $\mathrm{Sp}, \%$ & PLR \\
\hline Seo et al. ${ }^{21}$ & Korean 2015 & 567 & 71.6 & 7.8 & 0.77 & 71.2 & 0.40 & 73.9 & 2.70 \\
\hline \multirow[t]{2}{*}{ Jia et al. ${ }^{12}$} & China 2015 & 469 & 61.2 & 9.1 & 0.82 & 32.0 & 0.72 & 0.95 & 6.4 \\
\hline & & & & 4.7 & & 95 & 0.10 & 51 & 1.94 \\
\hline Goyal et al. ${ }^{22}$ & India 2013 & 357 & 23.2 & 6.0 & 0.84 & 82.0 & - & 67.0 & - \\
\hline \multirow[t]{2}{*}{ Chen et al. ${ }^{13}$} & China 2012 & 291 & 79.4 & 9.8 & 0.86 & & & 94.5 & 11.0 \\
\hline & & & & 5.0 & & 99.1 & 0.04 & & \\
\hline Kim et al. ${ }^{62}$ & Korea 2012 & 194 & 84.5 & 8.8 & 0.87 & 78.0 & 0.25 & 86.7 & 5.8 \\
\hline Cardoso et al. ${ }^{63}$ & France 2012 & 202 & 42.1 & 7.2 & 0.87 & 74.0 & 0.30 & 88.0 & 6.20 \\
\hline Verveer et al. ${ }^{64}$ & Netherlands 2012 & 125 & 53.5 & 6.0 & 0.85 & - & - & - & - \\
\hline \multirow[t]{2}{*}{ Viganò et al. ${ }^{14}$} & Italy 2011 & 125 & 52.8 & 9.4 & - & - & - & 96.0 & 14.0 \\
\hline & & & & 6.2 & - & 94.0 & 0.10 & - & - \\
\hline Degos et al. ${ }^{65}$ & France 2010 & 284 & 41.5 & 5.2 & 0.78 & 89.0 & 0.29 & 38.0 & 1.43 \\
\hline \multirow[t]{2}{*}{ Kim et al. ${ }^{17}$} & Korea 2010 & $\begin{array}{l}104 \\
(A L T \leq U L N)\end{array}$ & $\sim 90$ & 6.0 & - & 86.4 & 0.21 & 63.5 & 2.36 \\
\hline & & $\begin{array}{l}52(\text { ULN }< \\
A L T \leq 2 \text { ULN })\end{array}$ & $\sim 90$ & 8.9 & - & 73.9 & 0.21 & 75.0 & 2.96 \\
\hline Sporea et al. ${ }^{66}$ & Romania 2010 & 140 & 76.4 & 7.0 & 0.66 & 59.0 & 0.59 & 70.0 & 1.97 \\
\hline Marcellin et al. ${ }^{24}$ & France 2009 & 173 & 50.3 & 7.2 & 0.81 & 70.0 & 0.36 & 83.0 & 4.10 \\
\hline Wang et al. ${ }^{67}$ & $\begin{array}{l}\text { Taiwan, China } \\
2009\end{array}$ & 88 & NA & 8.0 & 0.86 & 80.0 & 0.26 & 77.0 & 3.50 \\
\hline
\end{tabular}

Abbreviations: ALT, alanine aminotransferase; AUROC, area under receiver operating characteristic curve; F, METAVIR fibrosis stage; NLR, negative likelihood ratio; PLR, positive likelihood ratio; Se, sensitivity; Sp, specificity; ULN, upper limit of normal; VCTE, vibration controlled transient elastography.

more than 200 patients. For exclusion of cirrhosis diagnosis, the suggested cutoffs with NLR of $<0.1$ have ranged between $8.4 \mathrm{kPa}$ and $11.0 \mathrm{kPa}$.

The cutoff of $9.1 \mathrm{kPa}$ suggested by Liang et al. ${ }^{25}$ was derived from a study cohort that included patients with abnormal bilirubin. However, abnormal bilirubin impairs the performance for cirrhosis detection, and a previous study has recommended bilirubin normalization as being important for improving VCTE performance. ${ }^{26}$ The cutoff of $9.4 \mathrm{kPa}$ with NLR of 0.02 suggested by Vigano et al. ${ }^{14}$ indicated that higher LSM with NLR near 0.1 may be more suitable for excluding diagnosis. Therefore, the suggested LSM of 10.6 $\mathrm{kPa}$ by Chen et al. ${ }^{26}$ and of $11.0 \mathrm{kPa}$ suggested by Marcellin et $a .^{24}$ derived from studies using normal bilirubin could be used for excluding cirrhosis diagnosis.

Thus, in antiviral treatment-naive patients with normal bilirubin and compensated CHB, LSM of $10.6 \mathrm{kPa}$ and $17.0 \mathrm{kPa}$ could be used as cutoffs for excluding and confirming cirrhosis diagnosis, respectively. For patients showing values corresponding to the grey zone between LSM 10.6-17.0 kPa, cutoffs of $10.6 \mathrm{kPa}$ for ALT <2 ULN and $12.7 \mathrm{kPa}$ for ALT >2 ULN could be applied for confirming diagnosis of advanced fibrosis (METAVIR $F \geq 3$ ) to indicate immediate antiviral treatment. $^{26}$

With dual cutoffs of LSM for cirrhosis detection, some patients would still be left undiagnosed. To address this issue, stepwise combinations of VCTE with other routine available markers, such as FIB-4, aspartate aminotransferase to platelet ratio index and red cell distribution width-platelet ratio, were applied to minimize the proportion of patients involved in the grey zone. ${ }^{25,27}$ The stepwise combination could also minimize the proportion of patients wrongly diagnosed as cirrhotic, due to fluctuating levels of ALT or hepatitis flares, which cause misleadingly high LSM even at 3-6 months after ALT normalization in patients with severe acute exacerbation of $\mathrm{CHB}^{28}$ To the contrary, cirrhotic patients with mild necro-inflammation would be characterized as having lower LSM, thereby resulting in false negative diagnosis. LSM-based index combined with other noninvasive parameters, such as albumin, international normalization ratio, and platelet and ultrasonic parameters, have been initially demonstrated as effective for abating this defect. ${ }^{23}$

\section{Monitoring treatment response}

The dynamic change of liver fibrosis during antiviral therapy is one of the critical endpoints of assessing treatment response, as fibrosis stages are associated with prognosis of $\mathrm{CHB}$. Use of potent antiviral agents has allowed the majority of $\mathrm{CHB}$ patients to obtain sustained virus suppression, following long-term therapy. Liver biopsy is, thus, not routinely performed in $\mathrm{CHB}$ patients that have treatment-suppressed HBV. On the other hand, large cohort studies have suggested that patients with liver fibrosis, and even cirrhosis, may achieve disease regression after 5 years of entecavir or tenofovir therapy. ${ }^{29,30}$

The need for monitoring fibrotic changes still exists, however. As a repeatable non-invasive method, VCTE is feasible for monitoring histological response in patients on antiviral therapy. Studies have reported significant decline in LSM after antiviral therapy, implicating potential regression of liver fibrosis in the patients. ${ }^{31-33}$ One issue that should be 
Liang X.E. et al: VCTE in chronic hepatitis B

Table 2. Diagnostic performance of VCTE for liver cirrhosis (METAVIR F4) in patients with chronic hepatitis B

\begin{tabular}{|c|c|c|c|c|c|c|c|c|c|}
\hline Author & Country/year & Patients, $n$ & $F=4, \%$ & $\begin{array}{l}\text { Cutoff } \\
\mathrm{kPa}\end{array}$ & AUROC & $\mathrm{Se}, \%$ & NLR & $\mathrm{Sp}, \%$ & PLR \\
\hline Seo et al. ${ }^{21}$ & Korean 2015 & 567 & 20.5 & 11.6 & 0.90 & 85.3 & 0.20 & 84.9 & 5.70 \\
\hline \multirow[t]{2}{*}{ Jia et al. ${ }^{12}$} & China 2015 & 469 & 12.2 & 8.2 & 0.90 & 95.0 & 0.07 & 69 & 3.03 \\
\hline & & & & 21.3 & & 40 & 0.63 & 95 & 8 \\
\hline Goyal et al. ${ }^{22}$ & India 2013 & 357 & 5.9 & 9.0 & 0.93 & 81.0 & 0.21 & 90.0 & 8.1 \\
\hline Kim et al. ${ }^{62}$ & Korea 2012 & 194 & 38.7 & 14.1 & 0.91 & 84.0 & 0.19 & 84.9 & 5.56 \\
\hline $\begin{array}{l}\text { Cardoso } \\
\text { et al. }\end{array}$ & France 2012 & 202 & 7.9 & 11.0 & 0.94 & 75.0 & 0.28 & 90.0 & 7.34 \\
\hline \multirow[t]{4}{*}{ Chen et al. ${ }^{26}$} & China 2012 & $\begin{array}{l}213 \text { (normal } \\
\text { bilirubin) }\end{array}$ & 20.7 & 10.6 & 0.90 & 93.2 & 0.09 & 75.7 & 3.90 \\
\hline & & & & 16.9 & & 59.1 & 0.45 & 94.2 & 10.2 \\
\hline & & $\begin{array}{l}93 \text { (abnormal } \\
\text { bilirubin) }\end{array}$ & 32.2 & 9.1 & 0.84 & 100 & 0 & 46.9 & 1.90 \\
\hline & & & & 29.2 & & 23.3 & 0.73 & 98.4 & 14.7 \\
\hline $\begin{array}{l}\text { Verveer } \\
\text { et al. }{ }^{64}\end{array}$ & $\begin{array}{l}\text { Netherlands } \\
2012\end{array}$ & 125 & 6.4 & 13.0 & 0.90 & - & - & - & - \\
\hline \multirow[t]{2}{*}{ Viganò et al. ${ }^{14}$} & Italy 2011 & 125 & 16.0 & 13.1 & - & - & - & 95.0 & 14.0 \\
\hline & & & & 9.4 & - & 98.0 & 0.02 & - & - \\
\hline Degos et al. ${ }^{65}$ & France 2010 & 284 & 10.2 & 12.9 & 0.85 & 51.7 & 0.52 & 92.9 & 7.33 \\
\hline \multirow[t]{2}{*}{ Kim et al. ${ }^{68}$} & Korea 2010 & $104(\mathrm{ALT} \leq \mathrm{ULN})$ & $\sim 47$ & 10.1 & 0.88 & 86.7 & 0.15 & 88.1 & $7.26-$ \\
\hline & & $\begin{array}{l}52 \\
(U L N< \\
A L T \leq 2 U L N)\end{array}$ & $\sim 47$ & 15.5 & - & 66.7 & 0.33 & 100 & $\propto$ \\
\hline Sporea et al. ${ }^{66}$ & Romania 2010 & 140 & 5.0 & 13.6 & 0.97 & 86.0 & 0.14 & 99.0 & 86 \\
\hline \multirow{2}{*}{$\begin{array}{l}\text { Marcellin } \\
\text { et al. }{ }^{24}\end{array}$} & France 2009 & 173 & 8.1 & 11.0 & 0.93 & 93.0 & 0.08 & 87.0 & 7.20 \\
\hline & & & & 18.2 & & 57.0 & 0.44 & 97.0 & 19.0 \\
\hline \multirow[t]{4}{*}{ Chan et al. ${ }^{16}$} & $\begin{array}{l}\text { Hong Kong, } \\
\text { China } 2009\end{array}$ & $\begin{array}{l}58 \\
\text { (normal ALT) }\end{array}$ & 26.0 & 9.0 & 0.96 & 100 & 0 & 88.0 & 8.60 \\
\hline & & & & 12.0 & & 60.0 & 0.42 & 95.0 & 12.9 \\
\hline & & $\begin{array}{l}98 \\
\text { (abnormal ALT) }\end{array}$ & 25.0 & 8.4 & 0.94 & 96.0 & 0.07 & 54.0 & 2.10 \\
\hline & & & & 13.4 & & 75.0 & 0.27 & 93.0 & 11.1 \\
\hline Kim et al. ${ }^{69}$ & Korea 2008 & 91 & 42.9 & 10.3 & 0.80 & 59.0 & 0.53 & 78.0 & 2.68 \\
\hline Oliveri et al. ${ }^{70}$ & Italy 2008 & 188 & 20.0 & 11.8 & 0.97 & 86.5 & 0.14 & 96.3 & 23.2 \\
\hline Wang et al. ${ }^{67}$ & $\begin{array}{l}\text { Taiwan, China } \\
2009\end{array}$ & 88 & NA & 10.0 & 0.89 & 85.0 & 0.17 & 88.0 & 7.20 \\
\hline
\end{tabular}

Abbreviations: ALT, alanine aminotransferase; AUROC, area under receiver operating characteristic curve; F, METAVIR fibrosis stage; NLR, negative likelihood ratio; PLR, positive likelihood ratio; Se, sensitivity; Sp, specificity; ULN, upper limit of normal; VCTE, vibration controlled transient elastography.

kept in mind is that LSM was validated initially for the assessment of fibrosis progression and not for regression; it is also important to consider that the absolute cutoffs of LSM were derived from studies of treatment-naive CHB patients. Whether these pre-treatment cutoffs still work well in HBVsuppressed patients has been challenged.

Wong et al. ${ }^{33}$ studied $71 \mathrm{CHB}$ patients undergoing paired liver biopsy, with VCTE performed before and at week 48 of antiviral treatment. Only $11 / 28(39 \%)$ patients who showed LSM decreased by $>30 \%$, and $1 / 2(50 \%)$ patients who showed LSM increased by $>30 \%$ had decreased and increased histological fibrosis stages, respectively. Up to $60 \%$ of patients had insignificant change in LSM. The author explained that decrease in serum ALT levels and hepatic necro-inflammation may lead to reduced LSM regardless of change in liver fibrosis at week 48, and that decrease in absolute LSM was unreliable as an indicator of liver fibrosis regression at week 48 . Thus, the obvious effect of ALT normalization on the interpretation of LSM changes should be taken into consideration in patients under treatment with antiviral therapy.

Later studies reported the longitudinal changes in LSM over relatively longer periods. One study found that LSM 
declined continuously and significantly from pretreatment baseline compared to treatment years 1,2 and 3 (medians: $12.9 \mathrm{kPa}, 7.5 \mathrm{kPa}, 6.5 \mathrm{kPa}$ and $4.7 \mathrm{kPa}$, respectively; all $P<0.05$ ). In addition, LSM was significantly decreased at year $2(P=0.0210)$ compared with that at year $1 .{ }^{34}$ In another study, median LSM decreased significantly from $14.3 \mathrm{kPa}$ at baseline to $7.3 \mathrm{kPa}$ after 3 years of entecavir treatment $(P<0.001)$. A higher baseline LSM was recognized as the single independent predictor of a significant decline in LSM on multivariate analysis. ${ }^{31}$

Taken together these reported findings suggest LSM as a useful tool for monitoring changes of liver fibrosis in CHB patients under antiviral treatment. However, without paired liver biopsies from before and after treatment for confirmation, the role of VCTE for liver fibrosis assessment in CHB patients undergoing long-term therapy with antivirals remains to be determined. Nonetheless, the decline in LSM, whether it results from regression of fibrosis, remission of necro-inflammation or both, can be regarded as a favorable predictor for treatment response and may also be associated with prognosis. ${ }^{35}$

\section{Predicting portal hypertension and esophageal varices}

As the end stage of chronic liver disease, the semi-quantitative diagnosis of liver cirrhosis (e.g. METAVIR F4) is a morphologic definition that does not allow for distinction between a fibrogenic process that is still in progress but potentially reversible, and a more advanced stage of the liver disease that becomes irreversible. Regarding the histologic features of cirrhosis that have not been traditionally linked to clinical outcomes, several authors have suggested performing subclassifications of compensated and decompensated cirrhosis based on substages. ${ }^{36}$ For example, compensated cirrhosis could be further refined as: (1) no portal hypertension (hepatic venous pressure gradient [HVPG] $<6 \mathrm{mmHg}$ ); (2) portal hypertension that is not clinically significant (HVPG between 6 and $10 \mathrm{mmHg}$ ); and (3) clinically significant portal hypertension (HVPG $>10 \mathrm{mmHg}$ or presence of collaterals); moreover, the sub-stages 1 and 2 (HVPG $<10 \mathrm{mmHg}$ ) would be considered as compensated cirrhosis without varices, while the sub-stage 3 (HVPG $>10 \mathrm{mmHg}$ ) would be considered as compensated cirrhosis with varices. In this scenario, HVPG (or varices) plays an important role in further discrimination of the pathological and functional states of the liver.

Considering the complexity of testing HVPG and screening esophagogastroduodenoscopy, LSM has been validated and recently recommended for predicting portal hypertension and esophageal varices. ${ }^{37,38}$ In the report of the Baveno VI Consensus Workshop, ${ }^{37}$ LSM $>15 \mathrm{kPa}$ is highly suggestive of compensated cirrhosis (or compensated advanced chronic liver disease), while LSM $\geq 20-25 \mathrm{kPa}$, alone or combined with platelet concentration and spleen size, is sufficient to rule-in clinically significant portal hypertension (HVPG $>10$ $\mathrm{mmHg}$ ). Furthermore, this report suggested that patients with LSM $<20 \mathrm{kPa}$ and with platelet count $>150,000$ have a very low risk of having varices that will require treatment, and can thus avoid the screening endoscopy.

In addition, LSM may not be accurate in predicting HVPG for decompensated cirrhosis cases in which, in addition to intrahepatic vascular resistance, there are complex hemodynamic changes. ${ }^{36}$ In a large CHB patient cohort study, poor correlation (Kendall's tau_b 0.236) was found between LSM and the size of esophageal varices. ${ }^{39}$ In a different, briefly described cohort, ${ }^{40}$ almost $40 \%$ of patients who had LSM $>20 \mathrm{kPa}$ or platelet count $<150,000$ and should undergo endoscopy actually did not have varices, resulting in low specificity and positive predictive value of the Baveno's VI criteria. To some extent, the role of LSM in predicting portal hypertension and esophageal varices mainly aims at ruling out, rather than ruling in, varices needing treatment and consequently avoiding unnecessary endoscopies (Fig. 1).

\section{Predicting disease progression and prognosis}

Disease progression in terms of development of HCC and hepatic decompensation is a severe clinical event associated with high mortality in patients with $\mathrm{CHB}$. Detection of patients at high risk of disease progression is critical for better management of $\mathrm{CHB}$. Histologic severity of liver fibrosis is known to be correlated with development of HCC and hepatic decompensation. ${ }^{3}$ Thus, based on the close relationship between LSM and histological fibrosis stage, many studies have validated that higher LSM value was associated with higher risk of disease progression.

In a consecutive cohort including 600 patients with $\mathrm{CHB}$, patient prognosis decreased as LSM increased. The 5-year overall survival was $97.1 \%$ in patients with LSM $<9 \mathrm{kPa}$ and $61.5 \%$ in patients with LSM $>20 \mathrm{kPa}$, and multivariate analysis showed that LSM had the highest hazard ratio with survival. ${ }^{41}$ Lee et al. ${ }^{42}$ stratified CHB patients into three groups according to LSM levels ( $<8.0 \mathrm{kPa}, 8.0-13.0 \mathrm{kPa}$, and $>13.0 \mathrm{kPa}$ ) when achieving complete virological response. Patients with LS value $>13.0 \mathrm{kPa}$ (hazard ratio: 12.336) or 8.0-13.0 kPa (hazard ratio: 8.832 ) were at significantly greater risk of developing liver-related events (any cirrhotic complication, HCC, and liver-related mortality) compared with those with LSM $<8.0 \mathrm{kPa}$. The potential of LSM for predicting clinical outcomes seems to be greater than that of liver biopsy, probably LSM is capable of assessing ongoing pathophysiological processes and functions that a biopsy cannot.

A recent study showed that baseline LSM, rather than histological fibrosis stage, was independently predictive of $\mathrm{HCC}$ development in patients with $\mathrm{CHB}$ when starting antiviral therapy. ${ }^{42}$ While CHB patients with LSM $\geq 13 \mathrm{kPa}$ were identified as having subclinical cirrhosis, LSM-defined subclinical cirrhosis was found to be independently associated with a risk of developing HCC, regardless of antiviral therapy (hazard ratio: 3.344 and 4.680 for with and without antiviral therapy, respectively). ${ }^{43}$

Given the association between LSM and the development of HCC, LSM-based algorithms have been developed and validated recently. Wong et al. ${ }^{44}$ showed that LSM-HCC score constructed from LSM, age, serum albumin and HBV DNA level was accurate for prediction of HCC in CHB patients, with AUROC 0.83 at year 3 and 0.89 at year 5 , which was higher than that of an ultrasound-based score, CU-HCC (AUROC, 0.75-0.81). Another LS-based prediction model, LSPS ( $=$ LS value $\times$ spleen diameter/platelet count) for HCC prediction that had been developed in $227 \mathrm{CHB}$ patients, was identified as capable of independent prediction of HCC development (hazard ratio: 1.541) after adjusting for age, serum albumin level and histological fibrosis stage. ${ }^{45}$ After incorporating LSM into the REACH-B scoring model (replacing the serum HBV DNA level), a better predictive performance was 
observed compared with a conventional approach (AUROC, 0.814 vs 0.629 , respectively). ${ }^{42,46}$ Though the combined use of LSM and FibroTest significantly predicted forthcoming liver-related events development, it had only a slight additional benefit compared to LSM or FibroTest alone. ${ }^{47}$

In order to continue to improve the LSM-based algorithms for long-term outcome prediction, several issues need to be taken into consideration. The LSM-based algorithms have been derived from specific populations, for example, a community-based population or a population with advanced liver disease. Thus, the application of these LSM-based algorithms in the general population needs further validation. Most of the algorithms use single LSM or LSM at baseline for outcome prediction, whereas dynamic monitoring of LSM may evaluate the risk of HCC development more efficiently. In a consecutive cohort study of 198 patients with chronic hepatitis C, follow-up LSM was performed at least 1 year after the initial LSM. During a median follow-up period of 47.8 months, HCC incidence was $7 / 13(53.8 \%)$ in patients with initial LSM $>12 \mathrm{kPa}$ and follow-up LSM $>12 \mathrm{kPa}, 1 / 16(6.3 \%)$ in initial LSM $>12 \mathrm{kPa}$ and follow-up LSM $<12 \mathrm{kPa}$ and $0 / 77$ in initial LSM $<12 \mathrm{kPa}$ and follow-up LSM $<12 \mathrm{kPa} .{ }^{48}$ The ontreatment LSM, as well as the dynamic changes of LSM for outcome prediction in $\mathrm{CHB}$ patients have not been well evaluated. ${ }^{49,50}$

\section{Confounding factors and limitations of VCTE}

Although VCTE is validated and has been widely applied in non-invasive evaluation of liver fibrosis and cirrhosis in various clinical settings, including in cases of $\mathrm{CHB}$, the confounding factors of LSM should always be taken into consideration when interpreting the clinical significance of LSM values. Factors that influence viscoelastic properties of the liver have been reported to potentially increase liver stiffness; these include the presence of acute exacerbation of hepatitis, extrahepatic arteriovenous or biliary obstruction, and congestive heart failure. ${ }^{51-54}$ Thus, EASL recommended that VCTE should not be used in patients with very high ALT levels $(>10 \times U L N) .{ }^{38}$ In addition, definitive evidence has also indicated that food intake affects the accuracy of LSM for the prediction of fibrosis stage; therefore, it is advised that VCTE be undertaken when the patient has been fasting for at least 2 hours. ${ }^{55,56}$ While at least 10 validated measurements and an interquartile range $<30 \%$ of the median value are required for a reliable LSM, an interquartile range $<21 \%$ is associated with higher accuracy of VCTE for fibrosis diagnosis. ${ }^{57}$ Last but not least, not all patients achieve reliable and successful LSM. Around $3 \%$ of patients have LSM failure and $>10 \%$ of patients have unreliable LSM. ${ }^{58,59}$

It has been reported that body mass index $\geq 28-30 \mathrm{~kg} / \mathrm{m}^{2}$, central obesity, ascites, narrow inter-rib spaces, advanced age and female sex were the risk factors of unreliable LSM and LSM failure. In case of no valid shot or unreliable measurement in obese patients, the $\mathrm{XL}$ probe could be used. Although the probes have comparable accuracy, lower liver stiffness cutoffs will be necessary when the XL probe is used to noninvasively assess liver fibrosis. ${ }^{60,61}$

\section{Conclusions}

VCTE is a noninvasive tool with high accuracy and reproducibility for effectively evaluating liver fibrosis stages in patients with CHB. LSM could also serve in helping to make clinical decisions for antiviral therapy, monitoring antiviral response, surveillance of liver-related complications and long-term outcomes. With the recommendations of LSM by clinical practice guidelines and consensus, the clinical application of LSM in patients with $\mathrm{CHB}$ has become widely developed and validated, but still needs further standardization.

\section{Acknowledgments}

This study was funded by the National Science and Technology Major Project (No. 2013ZX10002004 to YPC) and the Dean's Fund of Nanfang Hospital (No. $2015 \mathrm{C022}$ to XEL).

\section{Conflict of interest}

The authors have no conflict of interests related to this publication.

\section{Author contributions}

Involved in the study design and data collection (XEL, YPC), wrote the manuscript (XEL), guarantee of the manuscript, revised and finalized the manuscript (YPC). Both authors had full access to the final version of the paper and agreed to the submission.

\section{References}

[1] Ferlay J, Soerjomataram I, Dikshit R, Eser S, Mathers C, Rebelo M, et al. Cancer incidence and mortality worldwide: sources, methods and major patterns in GLOBOCAN 2012. Int J Cancer 2015;136:E359-E386. doi: 10.1002/ijc. 29210

[2] European Association For The Study Of The Liver. EASL clinical practice guidelines: Management of chronic hepatitis B virus infection. J Hepatol 2012;57:167-185. doi: 10.1016/j.jhep.2012.02.010.

[3] Ganem D, Prince AM. Hepatitis B virus infection-natural history and clinical consequences. N Engl J Med 2004;350:1118-1129. doi: 10.1056/NEJMra031087.

[4] World Health Organization. Guidelines for the prevention, care and treatment of persons with chronic hepatitis B infection. Available from: http://apps.who.int/iris/bitstream/10665/154590/1/9789241549059_eng.pdf, accessed March 2015

[5] Rockey DC, Caldwell SH, Goodman ZD, Nelson RC, Smith AD; American Association for the Study of Liver Diseases. Liver biopsy. Hepatology 2009; 49:1017-1044. doi: 10.1002/hep.22742.

[6] Manning DS, Afdhal NH. Diagnosis and quantitation of fibrosis. Gastroenterology 2008;134:1670-1681. doi: 10.1053/j.gastro.2008.03.001.

[7] Chen YP, Peng J, Hou JL. Non-invasive assessment of liver fibrosis in patients with chronic hepatitis B. Hepatol Int 2013;7:356-368. doi: 10.1007/ s12072-013-9439-y

[8] Friedrich-Rust M, Poynard T, Castera L. Critical comparison of elastography methods to assess chronic liver disease. Nat Rev Gastroenterol Hepatol 2016;13:402-411. doi: 10.1038/nrgastro.2016.86.

[9] Mehta SH, Lau B, Afdhal NH, Thomas DL. Exceeding the limits of liver histology markers. J Hepatol 2009;50:36-41. doi: 10.1016/j.jhep.2008.07.039.

[10] Chen YP, Liang XE. Non-invasive assessment of liver fibrosis: reduce or substitute the need for liver biopsy? Liver Int 2015;35:2483. doi: 10.1111/liv. 12824.

[11] Jaeschke R, Guyatt G, Sackett DL. Users' guides to the medical literature. III. How to use an article about a diagnostic test. A. Are the results of the study valid? Evidence-Based Medicine Working Group. JAMA 1994;271:389-391.

[12] Jia J, Hou J, Ding H, Chen G, Xie Q, Wang Y, et al. Transient elastography compared to serum markers to predict liver fibrosis in a cohort of Chinese patients with chronic hepatitis B. J Gastroenterol Hepatol 2015;30:756-762. doi: $10.1111 /$ jgh. 12840 .

[13] Chen YP, Liang XE, Zhang Q, Peng J, Zhu YF, Wen WQ, et al. Larger biopsies evaluation of transient elastography for detecting advanced fibrosis in patients with compensated chronic hepatitis B. J Gastroenterol Hepatol 2012;27:1219-1226. doi: 10.1111/j.1440-1746.2012.07122.x.

[14] Viganò M, Paggi S, Lampertico P, Fraquelli M, Massironi S, Ronchi G, et al. Dual cut-off transient elastography to assess liver fibrosis in chronic hepatitis 
B: a cohort study with internal validation. Aliment Pharmacol Ther $2011 ; 34$ : 353-362. doi: 10.1111/j.1365-2036.2011.04722.x.

[15] Liang XE, Chen YP, Zhang Q, Dai L, Zhu YF, Hou JL. Dynamic evaluation of liver stiffness measurement to improve diagnostic accuracy of liver cirrhosis in patients with chronic hepatitis B acute exacerbation. J Viral Hepat 2011; 18:884-891. doi: 10.1111/j.1365-2893.2010.01389.x.

[16] Chan HL, Wong GL, Choi PC, Chan AW, Chim AM, Yiu KK, et al. Alanine aminotransferase-based algorithms of liver stiffness measurement by transient elastography (Fibroscan) for liver fibrosis in chronic hepatitis B. J Viral Hepat 2009;16:36-44. doi: 10.1111/j.1365-2893.2008.01037.x.

[17] Kim SU, Kim DY, Park JY, Lee JH, Ahn SH, Kim JK, et al. How can we enhance the performance of liver stiffness measurement using FibroScan in diagnosing liver cirrhosis in patients with chronic hepatitis B? J Clin Gastroenterol 2010;44:66-71. doi: 10.1097/MCG.0b013e3181a95c7f.

[18] Castéra L, Bernard PH, Le Bail B, Foucher J, Trimoulet P, Merrouche W, et al. Transient elastography and biomarkers for liver fibrosis assessment and follow-up of inactive hepatitis B carriers. Aliment Pharmacol Ther 2011;33: 455-465. doi: 10.1111/j.1365-2036.2010.04547.x.

[19] Papatheodoridis GV, Manolakopoulos S, Margariti A, Papageorgiou MV, Kranidioti $\mathrm{H}$, Katoglou $A$, et al. The usefulness of transient elastography in the assessment of patients with $\mathrm{HBeAg}$-negative chronic hepatitis $B$ virus infection. J Viral Hepat 2014;21:517-524. doi: 10.1111/jvh.12176.

[20] Maimone S, Caccamo G, Squadrito G, Alibrandi A, Saffioti F, Spinella R, et al. A combination of different diagnostic tools allows identification of inactive hepatitis B virus carriers at a single time point evaluation. Liver Int 2017; 37:362-368. doi: 10.1111/liv.13246.

[21] Seo YS, Kim MY, Kim SU, Hyun BS, Jang JY, Lee JW, et al. Accuracy of transient elastography in assessing liver fibrosis in chronic viral hepatitis: A multicentre, retrospective study. Liver Int 2015;35:2246-2255. doi: 10.1111/liv.12808.

[22] Goyal R, Mallick SR, Mahanta M, Kedia S, Shalimar, Dhingra R, et al. Fibroscan can avoid liver biopsy in Indian patients with chronic hepatitis $B$. J Gastroenterol Hepatol 2013;28:1738-1745. doi: 10.1111/jgh.12318.

[23] Liang XE, Dai L, Yang SL, Zhong CX, Peng J, Zhu YF, et al. Combining routine markers improves the accuracy of transient elastography for hepatitis B cirrhosis detection. Dig Liver Dis 2016;48:512-518. doi: 10.1016/j.dld.2016. 02.002 .

[24] Marcellin P, Ziol M, Bedossa P, Douvin C, Poupon R, de Lédinghen V, et al. Noninvasive assessment of liver fibrosis by stiffness measurement in patients with chronic hepatitis B. Liver Int 2009;29:242-247. doi: 10.1111/j.1478-3231. 2008.01802.x.

[25] Liang XE, Zhong C, Huang L, Yang S, Zhu Y, Chen Y, et al. Optimization of hepatitis $\mathrm{B}$ cirrhosis detection by stepwise application of transient elastography and routine biomarkers. J Gastroenterol Hepatol 2017;32:459-465. doi: $10.1111 /$ jgh. 13475

[26] Chen YP, Liang XE, Dai L, Zhang Q, Peng J, Zhu YF, et al. Improving transient elastography performance for detecting hepatitis B cirrhosis. Dig Liver Dis 2012;44:61-66. doi: 10.1016/j.dld.2011.08.004.

[27] Chen YP, Hu XM, Liang XE, Huang LW, Zhu YF, Hou JL. Stepwise Application of FIB-4, Red cell distribution width-Platelet Ratio and APRI for Compensated Hepatitis B Fibrosis Detection. J Gastroenterol Hepatol 2017. doi: 10.1111/ jgh.13811.

[28] Wong GL, Wong VW, Choi PC, Chan AW, Chim AM, Yiu KK, et al. Increased liver stiffness measurement by transient elastography in severe acute exacerbation of chronic hepatitis B. J Gastroenterol Hepatol 2009;24:10021007. doi: 10.1111/j.1440-1746.2009.05779.x.

[29] Marcellin P, Gane E, Buti M, Afdhal N, Sievert W, Jacobson IM, et al. Regression of cirrhosis during treatment with tenofovir disoproxil fumarate for chronic hepatitis B: a 5-year open-label follow-up study. Lancet 2013;381: 468-475. doi: 10.1016/S0140-6736(12)61425-1.

[30] Chang TT, Liaw YF, Wu SS, Schiff E, Han KH, Lai CL, et al. Long-term entecavir therapy results in the reversal of fibrosis/cirrhosis and continued histological improvement in patients with chronic hepatitis B. Hepatology 2010;52:886893. doi: 10.1002/hep.23785.

[31] Kim MN, Kim SU, Kim BK, Park JY, Kim DY, Ahn SH, et al. Long-term changes of liver stiffness values assessed using transient elastography in patients with chronic hepatitis B receiving entecavir. Liver Int 2014;34:1216-1223. doi: 10.1111/liv.12377.

[32] Fung J, Lai CL, Wong DK, Seto WK, Hung I, Yuen MF. Significant changes in liver stiffness measurements in patients with chronic hepatitis $B$ : 3-year follow-up study. J Viral Hepat 2011;18:e200-e205. doi: 10.1111/j.13652893.2010.01428.x.

[33] Wong GL, Wong VW, Choi PC, Chan AW, Chim AM, Yiu KK, et al. On-treatment monitoring of liver fibrosis with transient elastography in chronic hepatitis $B$ patients. Antivir Ther 2011;16:165-172. doi: 10.3851/IMP1726.

[34] Osakabe K, Ichino N, Nishikawa T, Sugiyama H, Kato M, Kitahara S, et al. Reduction of liver stiffness by antiviral therapy in chronic hepatitis $B$. J Gastroenterol 2011;46:1324-1334. doi: 10.1007/s00535-011-0444-4.
[35] Kim JH, Kim MN, Han KH, Kim SU. Clinical application of transient elastography in patients with chronic viral hepatitis receiving antiviral treatment. Liver Int 2015;35:1103-1115. doi: 10.1111/liv.12628.

[36] Garcia-Tsao G, Friedman S, Iredale J, Pinzani M. Now there are many (stages) where before there was one: In search of a pathophysiological classification of cirrhosis. Hepatology 2010;51:1445-1449. doi: 10.1002/hep. 23478.

[37] de Franchis R; Baveno VI Faculty. Expanding consensus in portal hypertension: Report of the Baveno VI Consensus Workshop: Stratifying risk and individualizing care for portal hypertension. J Hepatol 2015;63:743-752. doi: 10.1016/j.jhep.2015.05.022.

[38] European Association for Study of Liver; Asociacion Latinoamericana para el Estudio del Higado. EASL-ALEH Clinical Practice Guidelines: Non-invasive tests for evaluation of liver disease severity and prognosis. J. Hepatol 2015;63:237-264. doi: 10.1016/j.jhep.2015.04.006.

[39] Chen YP, Zhang Q, Dai L, Liang XE, Peng J, Hou JL. Is transient elastography valuable for high-risk esophageal varices prediction in patients with hepatitis-B-related cirrhosis? J Gastroenterol Hepatol 2012;27:533-539. doi: $10.1111 / j .1440-1746.2011 .06889 . x$.

[40] Perazzo H, Fernandes FF, Castro Filho EC, Perez RM. Points to be considered when using transient elastography for diagnosis of portal hypertension according to the Baveno's VI consensus. J Hepatol 2015;63:1048-1049. doi: 10.1016/j.jhep.2015.06.036

[41] de Lédinghen V, Vergniol J, Barthe C, Foucher J, Chermak F, Le Bail B, et al. Non-invasive tests for fibrosis and liver stiffness predict 5-year survival of patients chronically infected with hepatitis B virus. Aliment Pharmacol Ther 2013;37:979-988. doi: 10.1111/apt.12307.

[42] Lee HW, Yoo EJ, Kim BK, Kim SU, Park JY, Kim DY, et al. Prediction of development of liver-related events by transient elastography in hepatitis B patients with complete virological response on antiviral therapy. Am J Gastroenterol 2014;109:1241-1249. doi: 10.1038/ajg.2014.157.

[43] Kim MN, Kim SU, Kim BK, Park JY, Kim DY, Ahn SH, et al. Increased risk of hepatocellular carcinoma in chronic hepatitis B patients with transient elastography-defined subclinical cirrhosis. Hepatology 2015;61:1851-1859. doi: 10.1002/hep.27735.

[44] Wong GL, Chan HL, Wong CK, Leung C, Chan CY, Ho PP, et al. Liver stiffnessbased optimization of hepatocellular carcinoma risk score in patients with chronic hepatitis B. J Hepatol 2014;60:339-345. doi: 10.1016/j.jhep. 2013.09.029.

[45] Shin SH, Kim SU, Park JY, Kim DY, Ahn SH, Han KH, et al. Liver stiffnessbased model for prediction of hepatocellular carcinoma in chronic hepatitis $B$ virus infection: comparison with histological fibrosis. Liver Int 2015;35: 1054-1062. doi: 10.1111/liv.12621.

[46] Jung KS, Kim SU, Song K, Park JY, Kim DY, Ahn SH, et al. Validation of hepatitis $B$ virus-related hepatocellular carcinoma prediction models in the era of antiviral therapy. Hepatology 2015;62:1757-1766. doi: 10.1002/hep. 28115.

[47] Park MS, Kim SU, Kim BK, Park JY, Kim DY, Ahn SH, et al. Prognostic value of the combined use of transient elastography and fibrotest in patients with chronic hepatitis B. Liver Int 2015;35:455-462. doi: 10.1111/liv.12678.

[48] Wang HM, Hung CH, Lu SN, Chen CH, Lee CM, Hu TH, et al. Liver stiffness measurement as an alternative to fibrotic stage in risk assessment of hepatocellular carcinoma incidence for chronic hepatitis $C$ patients. Liver Int 2013;33:756-761. doi: 10.1111/liv.12118.

[49] Kim BK, Oh HJ, Park JY, Kim DY, Ahn SH, Han KH, et al. Early on-treatment change in liver stiffness predicts development of liver-related events in chronic hepatitis B patients receiving antiviral therapy. Liver Int 2013;33: 180-189. doi: 10.1111/liv.12020.

[50] Kim MN, Kim SU, Park JY, Kim DY, Han KH, Chon CY, et al. Risk assessment of liver-related events using transient elastography in patients with chronic hepatitis B receiving entecavir. J Clin Gastroenterol 2014;48:272-278. doi: 10.1097/MCG.0b013e31829a7247.

[51] Sagir A, Erhardt A, Schmitt M, Häussinger D. Transient elastography is unreliable for detection of cirrhosis in patients with acute liver damage. Hepatology 2008;47:592-595. doi: 10.1002/hep.22056.

[52] Arena U, Vizzutti F, Corti G, Ambu S, Stasi C, Bresci S, et al. Acute viral hepatitis increases liver stiffness values measured by transient elastography. Hepatology 2008;47:380-384. doi: 10.1002/hep.22007.

[53] Millonig G, Reimann FM, Friedrich S, Fonouni H, Mehrabi A, Büchler MW, et al. Extrahepatic cholestasis increases liver stiffness (FibroScan) irrespective of fibrosis. Hepatology 2008;48:1718-1723. doi: 10.1002/hep.22577.

[54] Millonig G, Friedrich S, Adolf S, Fonouni H, Golriz M, Mehrabi A, et al. Liver stiffness is directly influenced by central venous pressure. J Hepatol 2010; 52:206-210. doi: 10.1016/j.jhep.2009.11.018.

[55] Mederacke I, Wursthorn K, Kirschner J, Rifai K, Manns MP, Wedemeyer H, et al. Food intake increases liver stiffness in patients with chronic or resolved hepatitis C virus infection. Liver Int 2009;29:1500-1506. doi: 10.1111/j. 1478-3231.2009.02100.x.

[56] Arena U, Lupsor Platon M, Stasi C, Moscarella S, Assarat A, Bedogni G, et al. Liver stiffness is influenced by a standardized meal in patients with chronic 
hepatitis C virus at different stages of fibrotic evolution. Hepatology 2013 ; 58:65-72. doi: 10.1002/hep.26343.

[57] Lucidarme D, Foucher J, Le Bail B, Vergniol J, Castera L, Duburque C, et al. Factors of accuracy of transient elastography (fibroscan) for the diagnosis of liver fibrosis in chronic hepatitis C. Hepatology 2009;49:1083-1089. doi: 10.1002/hep.22748.

[58] Wong GL, Wong VW, Chim AM, Yiu KK, Chu SH, Li MK, et al. Factors associated with unreliable liver stiffness measurement and its failure with transient elastography in the Chinese population. J Gastroenterol Hepatol 2011;26: 300-305. doi: 10.1111/j.1440-1746.2010.06510.x.

[59] Castéra L, Foucher J, Bernard PH, Carvalho F, Allaix D, Merrouche W, et al. Pitfalls of liver stiffness measurement: a 5 -year prospective study of 13,369 examinations. Hepatology 2010;51:828-835. doi: 10.1002/hep.23425.

[60] de Lédinghen V, Wong VW, Vergniol J, Wong GL, Foucher J, Chu SH, et al. Diagnosis of liver fibrosis and cirrhosis using liver stiffness measurement: comparison between $M$ and XL probe of FibroScan $®$. J Hepatol 2012;56: 833-839. doi: 10.1016/j.jhep.2011.10.017.

[61] Myers RP, Pomier-Layrargues G, Kirsch R, Pollett A, Duarte-Rojo A, Wong D, et al. Feasibility and diagnostic performance of the FibroScan XL probe for liver stiffness measurement in overweight and obese patients. Hepatology 2012;55:199-208. doi: 10.1002/hep.24624.

[62] Kim BK, Kim HS, Park JY, Kim DY, Ahn SH, Chon CY, et al. Prospective validation of ELF test in comparison with Fibroscan and FibroTest to predict liver fibrosis in Asian subjects with chronic hepatitis B. PLoS One 2012;7:e41964. doi: 10.1371/journal.pone.0041964.

[63] Cardoso AC, Carvalho-Filho RJ, Stern C, Dipumpo A, Giuily N, Ripault MP, et al. Direct comparison of diagnostic performance of transient elastography in patients with chronic hepatitis B and chronic hepatitis C. Liver Int 2012; 32:612-621. doi: 10.1111/j.1478-3231.2011.02660.x.
[64] Verveer $\mathrm{C}$, Zondervan $\mathrm{PE}$, ten Kate $\mathrm{FJ}$, Hansen $\mathrm{BE}$, Janssen $\mathrm{HL}$, de Knegt RJ. Evaluation of transient elastography for fibrosis assessment compared with large biopsies in chronic hepatitis B and C. Liver Int 2012;32:622-628. doi: 10.1111/j.1478-3231.2011.02663.x.

[65] Degos F, Perez P, Roche B, Mahmoudi A, Asselineau J, Voitot H, et al. Diagnostic accuracy of FibroScan and comparison to liver fibrosis biomarkers in chronic viral hepatitis: a multicenter prospective study (the FIBROSTIC study). J Hepatol 2010;53:1013-1021. doi: 10.1016/j.jhep.2010.05.035.

[66] Sporea I, Sirli R, Deleanu A, Tudora A, Popescu A, Curescu M, et al. Liver stiffness measurements in patients with HBV vs HCV chronic hepatitis: a comparative study. World J Gastroenterol 2010;16:4832-4837. doi: 10. 3748/wjg.v16.i38.4832.

[67] Wang JH, Changchien CS, Hung CH, Eng HL, Tung WC, Kee KM, et al. FibroScan and ultrasonography in the prediction of hepatic fibrosis in patients with chronic viral hepatitis. J Gastroenterol 2009;44:439-446. doi: 10. 1007/s00535-009-0017-y.

[68] Kim BK, Kim DY, Park JY, Ahn SH, Chon CY, Kim JK, et al. Validation of FIB-4 and comparison with other simple noninvasive indices for predicting liver fibrosis and cirrhosis in hepatitis B virus-infected patients. Liver Int 2010; 30:546-553. doi: 10.1111/j.1478-3231.2009.02192.x.

[69] Kim DY, Kim SU, Ahn SH, Park JY, Lee JM, Park YN, et al. Usefulness of FibroScan for detection of early compensated liver cirrhosis in chronic hepatitis B. Dig Dis Sci 2009;54:1758-1763. doi: 10.1007/s10620-008-0541-2.

[70] Oliveri F, Coco B, Ciccorossi P, Colombatto P, Romagnoli V, Cherubini B, et al. Liver stiffness in the hepatitis $B$ virus carrier: a non-invasive marker of liver disease influenced by the pattern of transaminases. World J Gastroenterol 2008;14:6154-6162. doi: 10.3748/wjg.14.6154. 